REALA-2007, núm. 304. RECENSIONES

\title{
IV. Recensiones
}


Sarasíbar Iriarte, M., El Derecho Forestal ante el Cambio Climático: las Funciones Ambientales de los Bosques, Aranzadi, Pamplona, 2007, 301 págs.

El desafío social, político y administrativo que supone el cambio climático, depende de las medidas que se adopten para la protección de los recursos ambientales; los bosques, en su doble función ambiental, como fuente de vida y sumidero de los gases de efecto invernadero, están llamados a convertirse en protagonistas de las actuaciones futuras. Este libro se ocupa de la función ambiental de los bosques y desarrolla a lo largo de un apartado introductorio y seis capítulos, que se completan con un apartado final dedicado a las conclusiones, el Derecho forestal ante los interrogantes que plantea el cambio climático.

«Evolución histórica de la política forestal y marco jurídico vigente» es el título del capítulo I de esta obra. La autora distingue varias etapas en la evolución histórica de la política forestal española. Hasta el siglo XVI, la política era principalmente de tipo penal, determinada por los abusos que se cometían y por la ausencia de claridad en la delimitación de los usos permitidos; la actividad administrativa de tipo penal fue menos intensa durante el reinado de los Reyes Católicos, pero siguió determinanda por la necesidad de favorecer a la ganadería.

En los siglos XVI y XVII, las disposiciones sobre los bosques estuvieron determinadas por el aprovechamiento de la madera para la construcción de buques, y por la aparición de una tímida política protectora con tintes conservacionistas. Durante el siglo XVIII, y primer tercio del XIX, el aumento de la población y de la demanda de madera, unido a la inexistencia de una auténtica conciencia forestal, al proceso de desamortización y a la propia inestabilidad política, dieron lugar a una política forestal deficitaria, y a la continua destrucción de bosques.

Las Ordenanzas generales de Montes elaboradas por Javier de Burgos en el año 1833, representan el inicio de una nueva etapa, caracterizada por la creación de una organización administrativa propia sobre montes, y por la creación de normas para la gestión racional de los montes. El gobierno de Mendizábal, con la redacción de la Ley de 1837 , que nacionalizó y expuso a pública subasta los bosques, dehesas y pastos de las comunidades religiosas, y con la creación del Cuerpo de Ingnieros de Montes, supuso un período determinante para la evolución posterior de esta materia.
Las leyes que se sucedieron en la segunda mitad del siglo XIX, establecieron los principios de la repoblación forestal y crearon las divisiones hidrológico-forestales, y el Patrimonio Forestal del Estado. Este primer apartado concluye con el estudio de la Ley de Montes de 1957, una norma que ha marcado la política forestal española durante un amplio período de tiempo, y cuya finalidad principal era la regulación del bosque como medio de producción, si bien presentaba algunas tímidas referencias ambientales

La participación de la Unión Europea en los foros internacionales sobre política forestal, ha estado determinada por dos elementos: de un lado, por la preocupación comunitaria sobre la situación forestal más allá de las fronteras de los Estados miembros, determinada por su deficiente producción forestal y la dependencia de las importaciones; y de otro lado, por las referencias del Protocolo de Kyoto, sobre su cumplimiento mediante la realización de actividades en terceros Estados, y la relevancia de las actividades forestales sobre el cambio climático.

El Tratado Constitutivo no incorpora referencia alguna sobre política forestal, lo que ha ocasionado que las iniciativas comunitarias que han sido adoptadas en este ámbito, han sido fundamentalmente desarrolladas en base a las competencias sobre medio ambiente. En el ámbito comunitario destaca la Estrategia Forestal Europea, y el Plan comunitario de los bosques contra la contaminación atmosférica, instrumentos que han puesto de manifiesto la relevancia de los bosques y su enorme potencial económico y ambiental, en el que destaca su influencia en la lucha contra la contaminación atmosférica.

La política forestal española se analiza desde una triple perspectiva, que se corresponde con las competencias que ostentan la Administración General del Estado, las Comunidades Autónomas y los Entes Locales. El artículo 45 CE y el esquema de distribución de competencias trazado por el texto constituticional, representaron una nueva etapa para el Dereho forestal español. La Ley de Montes de 2003 constituye el texto básico sobre el que se asienta la modernización de la legislación forestal que necesitaba nuestro ordenamiento, anclado en la regulación del año 1957.

Mediante esta norma se materializa la demanda social sobre conservación y protección de los bosques, con el fin de evitar el proceso de degradacíón al que se encuentran sometidos. Esta norma ha sufrido una destacada modificación en 2006, con el fin de corregir las deficiencias que habían sido detectadas; especial atención merece 
el tratamiento jurídico de la lucha contra incendios y las mejoras introducidas en el sistema de registro y catalogación de los montes. En lo que respecta al cambio climático, la Ley incorpora destacadas referencias sobre su configuración como un problema vinculado a las medidas forestales, así como el reconocimiento de la función ambiental de sumidero de los bosques.

El capítulo II se centra en el análisis del concepto y funciones de los bosques. En primer lugar, la autora analiza el significado de los términos monte y bosque, dos conceptos diversos que son empleados como sinónimos en la actual Ley de Montes, al igual que en buena parte de la normativa autonómica; esta ausencia de claridad terminológica, puede desembocar en errores y en una mala interpretación de la propia norma, como pone de manifiesto Sarasíbar Iriarte.

El siguiente apartado de este capítulo estudia las funciones económica, social y ambiental de los bosques. El reconocimiento jurídico de las funciones ambientales, la influencia de los bosques sobre el ciclo hidrológico, el suelo y la diversidad biológica, así como la concepción de los bosques como sumidero de carbono, se han ido incoporando de forma paulatina al Derecho forestal, donde la funciòn ecológica se ha convertido en el fin primordial. Esta nueva concepción ambiental conlleva una reinterpretación del derecho de propiedad sobre un espacio forestal; la función social de este derecho de propiedad adquiere una nueva dimensión, ya que debe atender a las múltiples funciones de los bosques, además de dar respuesta a las demandas de protección y conservación de estos espacios. La actual Ley de Montes se sitúa en esta perspectiva protectora e intervencionista, por parte de la Administración, en defensa de la propiedad forestal.

La función ambiental de los bosques como sumidero en el marco de la gestión forestal sostenible es objeto de análisis en el capítulo III. La consecución del desarrollo sostenible, como fin último del principio de integración ambiental, constituyen los elementos básicos sobre los que se desarrollan los primeros apartados de este capítulo, en los que la autora analiza la implantación de este principio en la política comunitaria, y su vinculación con el cambio climático. A partir de este análisis se desarrolla la función de sumidero de los bosques como principio y como función; la Convención Marco de las Naciones Unidas sobre el Cambio Climático de 1992, y su posterior desarrollo por el Protocolo de Kyoto en 1997, han reconocido la relevancia de los sumideros, como principio general, y como instrumento para la consecución de los compromisos de reducción de las emisiones contaminantes impues- tos a las Partes; la función de los bosques como sumidero ha sido reconocida por la vigente Ley de Montes, y en la legislación autonómica.

El capítulo IV analiza las medidas administrativas de fomento que han sido adoptadas para incentivar las actividades que persigan un desarrollo forestal sostenible, y que se encuentran previstas en la Ley de Montes. Sarasíbar Iriarte comienza este capítulo con el estudio de la actividad administrativa de ordenación, como pieza clave para una gestión forestal sostenible. La legislación vigente menciona como instrumentos de ordenación a los proyectos de ordenación y los planes dasocráticos; sin perjuicio de los referentes de ordenación más genéricos elaborados por la Administración General del Estado y las Comunidades Autonómas. Asimismo, la Ley de Montes también preve un régimen específico para aquellos espacios que requieran medidas de especial protección, con el fin de atender a sus características innatas.

La actividad de planificación que lleva a cabo la Administración a través de planes y programas, permite identificar las necesidades del sector, elaborar las líneas de actuación futura y actuar sobre la gestión y aprovechamiento de los bosques, por lo que también favorece el desarrollo de su función de sumidero. Las actividades limitadoras y sancionadoras completan el conjunto de medidas que son adoptadas por la Administración con el fin de incentivar la aplicación de los principios de sostenibilidad ambiental en la gestión y aprovechamiento de los bosques.

El capítulo V analiza el fomento como medida principal para la función de sumidero de los bosques. Mediante la actividad de fomento, la Administración anima al particular para que su labor se dirija hacia la consecución de fines de interés general, mediante el ofrecimiento de estímulos, principalmente económicos. La Ley de Montes reconoce la finalidad ambiental como objetivo prioritario de las medidas de fomento, e incorpora tres tipos de incentivos económicos: las subvenciones, los créditos y los incentivos por las externalidades ambientales. Este apartado se completa con las referencias al desarrollo de las medidas de fomento en la legislación forestal autonómica, previa distinción de las Comunidades Autónomas que han elaborado una ley forestal propia, y aquéllas otras que han desarrollado una normativa sobre fomento forestal, aún cuando no disponen de una ley forestal autonómica.

El segundo apartado de este capítulo atiende a la relevante vinculación entre el desarrollo rural y los espacios forestales. La Política Agrícola Común elaborada por la Unión Europea y las dispo- 
siciones comunitarias que la han desarrollado, han incorporado un conjunto de medidas para el desarrollo rural entre cuyos objetivos se encuentra la protección y conservación forestales. La autora se centra en el estudio de las medidas ruralistas, entre cuyas finalidades se encuentra la atención al cuidado del ambiente y la protección del entorno natural; y las medidas de tipo mixto, que incluyen la ayuda a la formación agroambiental y a la silvicultura, que presentan caracteres productivistas y ambientales.

El uso energético de la biomasa forestal es objeto de estudio en el capítulo VI. En lo que respecta a los residuos forestales, no existe una regulación específica al respecto, y la Ley de Montes, a pesar de emplear este término no lo define. Los residuos forestales forman parte de la biomasa, y su régimen se rige por lo dispuesto en la regulación jurídica general, principalmente en lo dispuesto en la Ley 10/1998, de residuos. La biomasa forestal representa una fuente energética alternativa al empleo de combustibles fósiles, lo que supone un paso más en la lucha contra el cambio climático. Desde las instituciones comunitarias y las autoridades españolas han elaborado diversas iniciativas que ponen de manifiesto la voluntad de fomentar el uso de la biomasa como una fuente energética; sin embargo son tímidos intentos, que aún no se han traducido en un desarrollo efectivo de las posibilidades de este recurso.

Esta obra destaca no sólo por la actualidad de la materia analizada, el cambio climático, sino también por la relación que establece entre esta grave preocupación ambiental y la función ambiental de los bosques. El exhaustivo estudio de la autora permite conocer la realidad actual del Derecho Forestal desde un punto de vista crítico, y con apartados específicos dedicados a la regulación comunitaria y a las políticas autonómicas sobre esta materia. La evolución de la legislación forestal desde una marcada perspectiva productivista hacia los objetivos ambientales que hoy la presiden, es una muestra del grado de concienciación ambiental que se ha alcanzado en nuestra sociedad, y que permite albergar esperanzas sobre la efectiva implantación de medidas frente al cambio climático.

M. ${ }^{a}$ Remedios Zamora Roselló Área de Derecho Administrativo Universidad de Málaga

RAZQUín LizÁRRAGA, J. A. y RuIZ DE APODACA EsPINOSA, A., Información, Participación y Justicia en
Materia de Medio Ambiente. Comentario sistemático a la Ley 27/ 2006, de 18 de julio, Aranzadi, Pamplona, 2007, 474 págs.

La obra se articula en cinco capítulos, además de un apartado introductorio y un anexo legislativo. El primero de los capítulos establece el marco social y jurídico en el que se elabora y desarrolla la Ley 27/2006. Con independencia de las distintas fuentes internacionales y europeas que han inspirado la elaboración de este texto, es evidente que refleja un cambio sustancial en la sociedad; ya que la ciudadanía exige ocupar un papel activo en los asuntos públicos.

El fenómeno participativo adquiere especial relevancia en un ámbito como el ambiental, en el que la pasividad de los poderes públicos ha convertido a la creciente concienciación ambiental de la sociedad, en la verdadera promotora e impulsora del derecho a un medio ambiente adecuado. El procedimiento administrativo se dirige, de esta forma, hacia una nueva etapa, en la que se ha convertido en un instrumento para la participación del ciudadano en los asuntos públicos.

Los textos internacionales han resultado determinantes para la efectiva implantación de los principios de información y participación pública. Si bien desde la Declaración de Estocolmo de 1972, las disposiciones internacionales han incoporado referencias a estas materias, será en la Declaración sobre el Medio Ambiente y el Desarrollo Sostenible, elaborada en Río de Janeiro en 1992, cuando se produce el reconocimiento conjunto de los derechos de información, participación y justicia ambientales, como elementos básicos de la denominada «democracia ambiental»; derechos posteriormente confirmados en la Declaración de Johannesburgo sobre Desarrollo Sostenible de 2002, y que han sido introducidos en los convenios ambientales que se han celebrado a nivel mundial y europeo.

El Convenio de Aarhus sobre el acceso a la información, la participación del público en la toma de decisiones y el acceso a la justicia en materia de medio ambiente, adoptado en el seno de la Comisión Económica para Europa de las Naciones Unidas, supuso un paso adelante en la implantación y desarrollo de los principios de la democracia ambiental y buena gobernanza. La dimensión social de los derechos ambientales adquiere una nueva significación con este Convenio jurídicamente vinculante, y que desarrolla la importancia de la participación de individuos y organizaciones para la consecución del desarrollo sostenible. 
La adopción de este Convenio ha influido en los criterios que inspiran la nueva normativa europea; si bien en el ámbito comunitario habían sido adoptadas disposiciones que regulaban la información y participación en materia de medio ambiente, se han incorporado nuevos principios de gobernanza y derechos fundamentales.

En el Derecho interno, la Constitución Española de 1978 supuso la incorporación de los principios de información, transparencia y participación pública, mientras que la entrada de nuestro país en la Comunidad Europea se tradujo en la adopción de las técnicas ambientales europeas. La regulación de las Comunidades Autónomas, expresión de sus competencias en materia de medio ambiente también ha contribuido al desarrollo e implantación de nuevas técnicas de protección ambiental, de los derechos ambientales y al establecimiento de la acción pública en áreas específicas.

Con el fin de transponer las Directivas comunitarias, desarrollar las disposiciones del Convenio de Aarhus, y subsanar las deficiencias de la regulación ambiental, se elaboró la Ley 27/2006, de 18 de julio, por la que se regulan los derechos de acceso a la información, de participación pública y de acceso a la justicia en materia de medio ambiente (LIPPJMA).

Razquin Lizarraga comienza el estudio de la Ley con un detallado análisis de las primeras etapas de la norma, en los estados de elaboración y tramitación parlamentaria. La distribución de competencias entre el Estado y las Comunidades Autónomas, se concreta en esta materia en la atribución al Estado de la legislación básica sobre protección del medio ambiente; mientras que las Comunidades Autónomas han asumido competencias de desarrollo legislativo en materia de medio ambiente y ecología, y competencia exclusiva sobre espacios naturales protegidos, según lo dispuesto en la legislación básica del Estado.

La siempre difícil delimitación competencial, se complica aún más en la materia de medio ambiente, como consecuencia de su carácter transversal, y su implicación en otras competencias sectoriales. El comentario de la jurisprudencia constitucional sobre esta materia, y el estudio de la delimitación competencial en el ámbito de evaluación de impacto ambiental, establecen un conjunto de criterios orientativos sobre los caracteres del carácter horizontal del medio ambiente.

La fundamentación competencial de la LIPPJMA se dispone en su disposición final tercera, y toma como referencia esencial la competencia estatal sobre la legislación básica en materia de medio ambiente, como regulación de mínimos que puede ser reforzada por las Comunidades Autónomas; sin perjuicio de la aplicación de otros títulos competenciales estatales que también resultan de aplicación. Especial atención merece la relación entre la LIPPJMA y la Ley de Régimen Jurídico de las Administraciones Públicas y del Procedimiento Administrativo Común, ya que el régimen específico desarrollado por la norma que analizamos, incorpora un nuevo sistema en materia ambiental que sólo prevé la aplicación del régimen general a falta de previsión específica, sin que puedan verse comprometidos los nuevos criterios de democracia ambiental.

Los derechos de acceso a la información, de participación en las decisiones ambientales y de acceso a los recursos administrativos y jurisdiccionales, se sitúan en la dimensión colectiva del artículo $45 \mathrm{CE}$, como expresión de derechos de carácter procedimental. Estos tres derechos procedimentales configuran el objeto de la LIPPJMA y permiten, a través de un único texto legal, el cumplimiento de las obligaciones derivadas de la ratificación del Convenio de Aarhus y de las Directivas comunitarias.

A continuación, el autor realiza un detallado análisis del catálogo de definiciones que se enuncian en el artículo 2 de la Ley, y que hacen referencia a los conceptos de público, personas interesadas, información ambiental, autoridades públicas, información que obra en poder de las autoridades públicas, información poseída en nombre de las autoridades públicas y solicitantes. Especial atención merece el concepto amplio de autoridad pública que adopta la LIPPJMA, que incluye en esta categoría a las entidades integrantes del sector público y otras personas de carácter privado; con el fin de garantizar el ejercicio de los derechos, las Administraciones públicas españolas tiene la obligación de elaborar listas de autoridades públicas, a las que puedan acceder todos.

El artículo 3 de la LIPPJMA reconoce los derechos de acceso a la información, participación pública y acceso a la justicia en materia de medio ambiente, y afirma su carácter específico en materia ambiental, así como su configuración como un instrumento para reforzar los derechos ya existentes. El sujeto activo de estos derechos son «todos», lo que permite la inclusión de las personas físicas y jurídicas, con una referencia específica a las organizaciones no gubernamentales de carácter ambiental.

Estos derechos son limitados, ya que su ejercicio se encuentra restringido por lo previsto en la propia Ley y en los principios de buena fe, la 
proscripción del abuso de derecho y la prohibición del uso antisocial de los derechos, condiciones previstas en el artículo 7 del Código Civil, al que se remite la LIPPJMA.

Los caracteres del derecho de acceso a la información ambiental son objeto de estudio del capítulo III. En el ámbito ambiental, la información desempeña un papel esencial para la concienciación social, a la vez que asegura la participación efectiva de los ciudadanos en los asuntos ambientales. Sin embargo, la efectividad práctica de este derecho exige una normativa reguladora de desarrollo, así como los medios personales y materiales indispensables para su implantación. La regulación de la Ley se inicia con un precepto dedicado a las obligaciones generales de las autoridades públicas en materia de información ambiental, que se completa con las referencias a los caracteres que rigen su difusión. El acceso a la información se constituye como un deber de suministrar la información por parte de las autoridades públicas, y un derecho de los ciudadanos a acceder a la misma previa solicitud.

La LIPPJMA establece una serie de exigencias procedimentales mínimas y básicas sobre las solicitudes de información ambiental, remitiéndose a los procedimientos establecidos a tal efecto sobre la tramitación y resolución de las peticiones de acceso a la información ambiental. Las excepciones sobre divulgación de la información requerida se organizan en dos bloques diferenciados, que permiten distinguir aquellas vinculadas a cuestiones procedimentales o de confidencialidad de las comunicaciones internas de las autoridades públicas. La referencia a la contraprestación económica, incluye la previsión de la Ley sobre las condiciones en que pueden ser exigidas tasas y precios públicos y privados, por recibir la información solicitada. Este capítulo finaliza con un apartado dedicado a la legislación autonómica, incluyendo el estudio de las normas sectoriales y las referencias organizativas que han desarrollado las Comunidades Autónomas en ejercicio de sus competencias sobre información ambiental.

El capítulo IV analiza el derecho de participación pública en asuntos de carácter ambiental. La participación pública es un pilar básico del sistema democrático, y de especial relevancia en el campo ambiental en lo relativo a la creación y ejecución de la normativa específica. Al igual que en otras materias, la LIPPJMA desarrolla las previsiones comunitarias, de la Directiva 2003/35/ $\mathrm{CE}$, y las internacionales, previstas en el Convenio de Aarhus; el resultado es la materialización del derecho a través de tres niveles de participación: las decisiones sobre actividades específicas, los planes y programas y las disposiciones de carácter general, y el Consejo Asesor de Medio Ambiente.

En lo que respecta al primer nivel de participación, Ruiz de Apodaca Espinosa, pone de manifiesto los caracteres de la participación en la evaluación de impacto ambiental de proyectos, en el procedimiento de autorización ambiental integrada, en el campo de la biotecnología, y en otras autorizaciones ambientales como las previstas en la normativa sobre aguas, residuos y actividades molestas, insalubres, nocivas y peligrosas. La LIPPJMA regula una serie de garantías comunes al segundo nivel de participación, a la participación pública en planes, programas y normas, que deben ser aseguradas por las Administraciones públicas, y constituyen un derecho para sus titulares; a continuación se exponen los caracteres propios de la participación en planes y programas, así como el ámbito de aplicación de la Ley en lo que respecta a la participación en las disposiciones de carácter general relacionadas con el medio ambiente. Este apartado se completa con las referencias a la participación orgánica a través del Consejo Asesor de Medio Ambiente y en los Consejos asesores de medio ambiente autonómicos.

El capítulo V analiza el derecho de acceso a la justicia y a la tutela administrativa en asuntos medioambientales, y su estudio comienza con las previsiones del Convenio de Aarhus y de las disposiciones comunitarias sobre esta materia. En este ámbito el autor considera que la regulación de la LIPPJMA es muy breve y poco novedosa. Uno de los aspectos más destacado en esta materia es la regulación de la acción pública circunscrita a las organizaciones no gubernamentales ambientales, y limitada por una serie de previsiones restrictivas. De esta forma, la Ley no admite la acción pública en materia ambiental con carácter general a todos los ciudadanos; a diferencia de un buen número de disposiciones autonómicas ambientales, que han optado por el reconocimiento de la acción popular, sin distinción entre personas jurídicas o físicas.

La significativa aportación de esta obra radica, no sólo en el detallado estudio de los preceptos de la LIPPJMA, sino también en el análisis comparativo de las aportaciones realizadas por el Convenio de Aarhus y las disposiciones comunitarias elaboradas en este ámbito. Este libro se convierte de esta forma en una obra de referencia en lo que respecta a la Ley $27 / 2006$, pero también en relación a numerosos temas colaterales que aparecen analizados en detalle a lo largo de todo el texto; con especial atención a la abundante jurisprudencia comentada que se incluyen en 
todos los apartados, y que permite establecer un marco de conexión entre la materialización de los derechos de información, participación y justicia en materia de medio ambiente, así como en otros ámbitos. La implicación de la ciudadanía en el desarrollo de las políticas ambientales adquiere una nueva perspectiva con las disposiciones internacionales, comunitarias y nacionales vigentes; ante este panorama, sólo resta que las autoridades competentes utilicen el texto que hemos comentado como libro de cabecera para la efectiva implantación de los derechos ya reconocidos.

M. ${ }^{a}$ Remedios Zamora Roselló Área de Derecho Administrativo Universidad de Málaga

\section{AA.VV., ORduña Rebollo, EnRIQUe (coord.), Valladolid en América. El municipalismo y su proyección ibe- roamericana, Ciudad Argentina, Buenos Aires-Madrid 2007. 327 pp.}

Esta obra recoge las ponencias emanadas del Seminario que, con motivo del V Centenario del fallecimiento de Cristóbal Colón, llevaron a cabo conjuntamente la Organización Iberoamericana de Cooperación Intermunicipal (OICI) y el Ayuntamiento de Valladolid, durante los días 25, 26 y 27 de octubre de 2006. El título de este Seminario es el mismo que el de este libro, cuyo contenido pluridisciplinar gira en torno a dos grandes bloques temáticos, distintos, pero complementarios; por un lado, cuestiones relativas a urbanismo, y por otro, a municipalismo y administración local, amén de otros temas colaterales a los que constituyen el eje del libro. Así pues, dada la naturaleza del mismo, como resultado de un encuentro en torno a una cuestión que puede contemplarse desde diferentes puntos de vista, el conjunto de aportaciones reunidas reviste una cierta heterogeneidad.

La premisa de la que parten los distintos estudios es la consideración de la ciudad de Valladolid como paradigma en el desarrollo urbano y municipal de las ciudades que surgieron en el Nuevo Mundo desde el momento inicial de la época del descubrimiento y colonización. Valladolid no sólo constituyó un modelo de ordenamiento territorial y urbanístico, sino también un modelo de organización municipal que se proyectó en Iberoamérica en el espacio y en el tiempo, primero mediante la administración virreinal y posteriormente a través del surgimiento de las nuevas repúblicas americanas. La ciudad de Va- lladolid, así como otras ciudades españolas del siglo XVI, estaba impregnada de las ideas humanistas en lo que a urbanismo y municipalismo se refiere; en lo primero, las nuevas concepciones urbanísticas procedentes de Italia calaron en los municipios españoles, en lo segundo, el referente ofrecido por las pequeñas repúblicas o ciudadesestado italianas se funde con la tradición de autonomía municipal existente en la Corona de Castilla desde la época de la repoblación, ya desde el siglo XII, donde a partir de pequeños núcleos rurales, se fueron desarrollando comunidades urbanas con amplias competencias municipales, en palabras de Enrique ORDUÑa REBOLlo, coordinador de este libro, un auténtico «señorío concejil», plasmadas en los fueros otorgados por la monarquía, que dos siglos más tarde se tradujo en un considerable desarrollo económico y social. Toda esta concepción del municipio fue exportada a América y constituyó una parte importante del legado cultural llevado por los colonizadores.

El volumen se articula, como se ha indicado anteriormente, de forma heterogénea en trece ponencias, cada autor aborda una cuestión enmarcada dentro del contexto general de la obra, coordinada, como se ha dicho, por Enrique ORDUÑA REBOLLo. Se abre con la presentación realizada por Francisco Javier LEÓN DE LA RIVA, Alcalde de Valladolid y Presidente de la OICI, en la que se subraya «la vocación municipalista iberoamericana de este Ayuntamiento», así como la implicación de la OICI en la «difusión y defensa del municipalismo iberoamericano». A continuación haremos una sucinta reseña del contenido de cada uno de los estudios.

Tras esta breve presentación, Luis Miguel ENCISO RECIO, Catedrático de Historia Moderna, presenta la ponencia titulada «La Valladolid en la que murió Colón», en la que primeramente hace un somero estudio del panorama socio-económico, el desarrollo urbano, las formas culturales, el estilo de vida y el gobierno municipal de Valladolid a comienzos del siglo XVI; seguidamente se centra en los últimos años de la vida del Almirante, de su paso por Sevilla, Segovia, Salamanca y Valladolid, las ciudades que le acogieron en esos momentos de su vida, de su controvertida relación con la Corona, en defensa de sus pretendidos derechos y la recuperación de sus títulos y privilegios tras su fallida experiencia de gobernador de los territorios descubiertos, hasta su fallecimiento en la ciudad del Pisuerga.

Seguidamente, Laura SAN MARTINO, Doctora en Historia, aborda un estudio titulado «La autonomía: una variable histórica de las ciudades». Se analiza la cuestión del poder autonómico ciuda- 
dano como algo consustancial a la ciudad misma, una facultad que va a generar sus propias instituciones locales. Tal competencia se traduce en una serie de potestades que configuran la soberanía local; de esta manera, partiendo de dicho poder autonómico municipal, las ciudades pueden conformar unidades territoriales y jurisdiccionales de rango superior, como las provincias, así, los sistemas federales de organización política tienen sus raíces en regímenes cívico-municipales. Además, el poder autonómico de las ciudades también se manifiesta en el conjunto de atribuciones y facultades para celebrar pactos institucionales, gestionar los servicios públicos locales, llevar a cabo el ordenamiento territorial y urbanístico, realizar el diseño cultural de la ciudad, desarrollar su tejido industrial, establecer las pautas de protección ambiental u organizar los cuerpos de seguridad local. Todas estas cuestiones son tratadas una por una y de forma pormenorizada por la autora.

Ana María FEIJóo CASADo, Directora del Archivo Municipal de Valladolid, nos ofrece en su aportación titulada «Riqueza documental colombina y americana en los Archivos de Valladolid» una aproximación al conocimiento del patrimonio documental existente en los archivos vallisoletanos. En cuanto a fondos y volumen la ciudad de Valladolid custodia una buena parte del patrimonio documental de los archivos españoles. En primer lugar, la autora describe los distintos archivos que hay en Valladolid, para seguidamente exponer la documentación que en ellos se halla. Se expone pormenorizadamente la documentación colombina existente en el Archivo General de Simancas, uno de los más importantes del mundo como fuente para el estudio de la historia moderna, y se examina someramente la que se encuentra diseminada en las distintas secciones y series. Más adelante trata también sobre la documentación del Archivo de la Real Chancillería de Valladolid, y de manera más puntual, la del Archivo Municipal de Valladolid, la del Archivo Histórico Provincial, la del Archivo Universitario de Valladolid y la del Archivo General Diocesano.

Enrique ORduña Rebollo, Secretario General de la OICI, en su estudio titulado «El modelo urbanístico de Valladolid en 1561 y su proyección en la ciudad iberoamericana» lleva a cabo un análisis sobre la ciudad de Valladolid en la segunda mitad del siglo XVI, momento en el que se puede considerar a la villa como el corazón de la Monarquía Hispana. Primeramente, a modo de introducción, se describe someramente la ordenación urbana de la ciudad, destacando la importancia de la plaza como elemento urbanístico. En segundo lugar se examinan sus instituciones y su componente social y urbano. A continuación se estudia la transformación urbanística acaecida en Valladolid en época de Felipe II, tras el incendio de 1561; entre esta fecha y 1573 se producen los acontecimientos que constituyen el núcleo central de este trabajo. Dicha actuación urbanística estuvo regulada por las nuevas Ordenanzas redactadas a tal efecto, que tuvieron como consecuencia el nuevo modelo urbanístico vallisoletano. A modo de conclusión, se puede decir, en palabras del autor, que este diseño de planificación urbana, impulsada por iniciativa real, constituyó el primer proyecto urbanístico integral de la Edad Moderna en ciudades españolas, pues sirvió como referencia para la planificación posterior en otras ciudades y territorios, tanto en España como en el Nuevo Mundo.

Germán Rueda HeRnanz, Catedrático de Historia Contemporánea, en su ponencia «La transformación urbana de Valladolid por el proceso desamortizador» estudia la transformación urbanística sufrida por la ciudad a lo largo del siglo XIX. Tras pasar revista al patrimonio urbano desamortizado, expone las modificaciones urbanísticas que se produjeron como consecuencia de la desamortización.

Laura SERRANo Blanco, Doctora en Historia Contemporánea, con su estudio titulado «La influencia de la crisis del 98 en el pensamiento regeneracionista y sus propuestas de reforma local» trata sobre el influjo del movimiento regeneracionista en el desarrollo local. Desglosa su intervención en dos partes, en primer lugar, expone cual es el panorama intelectual español en los momentos previos al 98. Analiza la evolución política española que arranca desde 1868 y las nuevas corrientes de pensamiento (krausismo y positivismo fundamentalmente) que se difunden por el país, encarnada en la minoría intelectual que Azorín definió como «los últimos erasmistas españoles». Estos inician un proceso de autorreflexión sobre la identidad nacional; en este grupo hay que incluir a los integrantes de la Institución Libre de Enseñanza y a otros intelectuales, entre los que destaca el autor vallisoletano de adopción Ricardo Macías Picavea. Se esboza un semblante de su pensamiento a través de su actividad política y periodística, destacando su propuesta de regeneración local anterior al 98, en la que hay que resaltar su defensa de la autonomía municipal y una relativa descentralización de poderes; a lo que hay que agregar su preocupación por la enseñanza y la salud e higiene pública. En segundo lugar, a modo de conclusión, realiza una reflexión acerca del significado histórico de la crisis de 1998, en la que resalta la crisis de la conciencia colectiva del país, así como la quiebra de 
la hegemonía ideológica de la oligarquía en el poder.

Armando Rodríguez García, Catedrático de Derecho Administrativo, se encarga en su ponencia titulada «La intermunicipalidad y el despertar del municipalismo iberoamericano» de encuadrar en la realidad iberoamericana el ideal de municipio que llevaron consigo los colonizadores españoles. Estos, como se ha dicho anteriormente, aplicaron en el Nuevo Mundo el concepto que de municipio existía en la Península Ibérica desde las épocas de la repoblación, en torno al año mil. El autor analiza como esta concepción del municipio arraigó en ultramar mediante el traslado de las instituciones locales peninsulares a tierras americanas, cómo se desarrollaron y cómo supusieron un elemento de integración primordial, constituyendo la clave del proceso de poblamiento y colonización. A continuación evalúa la trascendencia de la instancia municipal en el proceso político de surgimiento de las repúblicas americanas, para terminar haciendo una valoración del intermunicipalismo iberoamericano.

D. Roberto Dromi, Catedrático de Derecho Administrativo, procede en su intervención que lleva por título «El sistema de la ciudad» a hacer un análisis sobre la interrelación existente entre la organización local en un mundo global y la calidad de vida urbana resultante de la gestión local. Según indica el autor, la infraestructura de la ciudad es un condicionante esencial para su desarrollo y calidad de servicios públicos municipales, de la excelencia de estos dependerá dicha calidad de vida urbana y la legitimación política.

Valentín MERINo EsTRADA, Secretario General del Ayuntamiento de Valladolid, en su contribución titulada «Valladolid en la doctrina municipalista de los Congresos Iberoamericanos de Municipios» hace un repaso de los congresos iberoamericanos de municipios promovidos por la OICI, exponiendo su filosofía y objetivos básicos, que se pueden concretar en la defensa de la democracia y de la autonomía local, así como en el intento de conseguir actuaciones conjuntas de cooperación, basadas en la idea de intermunicipalidad. Seguidamente expone la doctrina emanada de dichos Congresos que resume en tres consideraciones: valoración del municipio como escenario en el que se gesta y desarrolla la cultura democrática y la participación ciudadana, visión del municipio como gestor de servicios públicos y concepción del mismo como organización estratégica. Termina su intervención señalando las aportaciones que desde Valladolid se han llevado a cabo.
Rosa Isabel Hernández del CAMPo, Concejala del Ayuntamiento de Valladolid, en su ponencia «La cooperación intermunicipal iberoamericana del Ayuntamiento de Valladolid» pone de manifiesto la política municipal de cooperación para el desarrollo que este Ayuntamiento lleva a cabo con países en desarrollo en general y con Iberoamérica en particular. En primer lugar, pasa revista al pasado y presente de la cooperación del Ayuntamiento de Valladolid con América Latina. En segundo lugar, esboza el futuro de la cooperación en dicho Ayuntamiento; apuntando, por un lado, los referentes teóricos, que quedan plasmados en un cambio en la concepción de desarrollo, que se traduce en el tránsito de un modelo de cooperación a un modelo de solidaridad; y, por otro, la líneas de acción para elaborar un plan estratégico municipal para la cooperación internacional.

Tras esta intervenciones, Fernando Rubio BALlestero, Consejero Delegado de Presidencia del Ayuntamiento de Valladolid, en la «Introducción a la Mesa Redonda: los retos de la ciudad en el siglo XXI» señala la perspectiva del modelo de ciudad que se configura en el nuevo siglo. Esto se plasma en la universalización de la gran ciudad dentro de un mundo globalizado. En palabras del autor, «el reto de la ciudad en el siglo XXI es enfocar un despliegue integral de una visión conjunta de la misma, que se adapte a los continuos cambios acontecidos en su entorno». Seguidamente, Carlos BARó PAzos, Ingeniero de Caminos, Canales y Puertos, en la «Continuación a la Mesa Redonda: los desafíos que plantea la transformación urbana futura de Valladolid» pone de manifiesto la influencia que las infraestructuras del transporte van a tener en el desarrollo ulterior de la urbe, evaluando el impacto en la economía y en el funcionamiento de la ciudad.

El volumen se cierra con las palabras de Francisco Javier LEÓN DE LA RIVA, Alcalde de Valladolid, en su intervención titulada «Conferencia de clausura: Valladolid y el municipalismo del siglo XXI», donde reitera y desarrolla las ideas vertidas en la presentación, enfatizando la vocación municipalista de la ciudad de Valladolid, paralela a su condición universalista. Se aboga por un fortalecimiento del municipalismo como pilar básico en el siglo entrante que garantice la convivencia y la participación ciudadana; en definitiva, por la promoción del desarrollo local dentro de un mundo global, para lo cual es imprescindible la cooperación intermunicipalista a nivel mundial.

José Manuel Valle VEGA Licenciado en Geografía e Historia Universidad Complutense de Madrid 
GÉRARD MARCOU, El alcance y la naturaleza de las competencias de las Entidades Locales en los Estados miembros del Consejo de Europa. Informe para el Comité Director para la Democracia Local y Regional del Consejo de Europa (C.D.R.L). Traducción de José Manuel Rodríguez Álvarez. Ministerio de Administraciones Públicas. Madrid, 2006, 145 págs.

Este libro, como muy bien señala en la presentación su traductor RoDRígUEZ ÁlvAREZ, viene a cubrir una laguna existente, no sólo en España sino también en Europa, sobre la cuestión de las competencias locales. No existía, a pesar de ser éste uno de los temas fundamentales del debate sobre los gobiernos locales desde los años noventa, estudios rigurosos de las competencias locales desde una perspectiva comparada, que aportasen luz sobre los modelos o sistemas competenciales existentes, partiendo de un análisis realista, institucionalista y estructural.

El abordar las competencias locales desde una perspectiva más compleja y sistemática que superase los esquemas meramente formales y descriptivos y que permitiera trabajar con mayor rigor y eficacia al Comité Director para la Democracia Local y Regional del Consejo de Europa se convirtió para este Comité, desde 2002, en una de sus prioridades. Para realizar el estudio se eligió a uno de los más importantes y prestigiosos especialistas del estudio comparado de los gobiernos locales en Europa, el profesor de la Universidad Paris I, GÉrARD MARCOU ${ }^{1}$, lo que demuestra el gran interés del Comité por esta cuestión. El profesor MARCOU reúne unas condiciones excepcionales para un estudio de esta clase ya que a sus amplios conocimientos, tanto teóricos como prác- ticos, en el campo de los estudios comparados de los gobiernos locales une el hecho de tratarse de un políglota que domina numerosas lenguas (inglés, francés, alemán, ruso, español, polaco), lo cual es importante en un trabajo como éste en el que es un elemento importante el análisis de los términos esenciales usados.

El objetivo del estudio era proponer un nuevo enfoque comparado de las competencias de las entidades locales, concentrándose en el primer nivel local, en los Estados del Consejo de Europa mediante el análisis de la situación concreta de una selección de Estados miembros, con el fin de deducir unos principios y un esquema comparativo que puedan ser aplicados, después de su verificación, corrección y validación, a otros Estados. Con ello se facilitaría a las autoridades nacionales apreciar la situación de su Estado, con respecto a la de los otros Estados miembros del Consejo de Europa, y en particular de sus vecinos, que no son siempre mejor conocidos que los Estados más alejados. Además se esperaba poder clasificar los países en diferentes categorías basadas en un conjunto de características significativas, algo que no pudieron conseguir; en cambio, nos proponen un esquema de análisis y evaluación de las competencias locales, que deja un espacio a la dinámica de los sistemas de administración local de los diferentes países.

La muestra de los países la hicieron a partir del conocimiento sobre los modelos de administración local de los mismos ${ }^{2}$, de forma que se garantizase la consideración de experiencias nacionales bastante variadas y contrastadas para que las conclusiones no estuvieran condicionadas por la referencia preponderante a un número de casos demasiado pequeño, de hecho demasiado singulares con respecto al resto de Europa. A las autoridades nacionales de los países seleccionados ${ }^{3}$ : Alemania, España, Francia, Hungría, Italia, los Países Bajos, Polonia, Portugal, Inglaterra y Suecia les enviaron un cuestionario ${ }^{4}$ para que lo respon-

\footnotetext{
${ }^{1}$ Gerard MARCoU es profesor de la Universidad I de París y Director del Grupo de Estudios sobre la Administración Local (GRALE) integrado en el Centro Nacional de Investigaciones Científicas (CNRS) de Francia. Ha participado en numerosos estudios y proyectos relacionados con la descentralización en numerosos países europeos, siendo con los profesores H. Wollmann y A. Lucarelli los redactores del informe sobre el primer borrador del Libro Blanco para la Reforma del Gobierno Local en España, realizado por encargo del Consejo Europa a petición de la representación española..

${ }^{2}$ Los países seleccionados son una muestra representativa de las diferencias tradicionales en los regímenes locales europeos: por una parte, la de Estados que han realizado una importante reforma del mapa local, para reducir el número de municipios (como Suecia, Alemania Occidental, el Reino Unido y los Países Bajos) y los que no (como España, Italia y Francia); y por otra parte, los que han atribuido a las entidades locales un papel muy importante en la gestión de los servicios más relevantes del Estado de bienestar (como Suecia o el Reino Unido) y los que no (como España o Italia).

${ }^{3}$ Todos estos países pertenecen no sólo al Consejo de Europa, sino también a la Unión Europea.

${ }^{4}$ Además de los cuestionarios, el autor previó misiones de estudio en tres países (Hungría, Países Bajos y Suecia) para recoger una información más completa y actual. Estos países fueron escogidos en función del interés particular de su sistema de administración local y del hecho de que la información sobre ellos estaba mucho menos representada en la literatura internacional.
} 
dieran, pero tres de ellos no respondieron (Alemania, Francia y Polonia), eliminándose de la investigación Polonia del cual, a diferencia de los otros dos casos, el autor no tenía posibilidad de conseguir información relevante. Además se realizaron viajes de estudio a tres países (Suecia, Hungría y Países Bajos) para recoger una información más completa y más actual.

Sobre esta información, MARCOU elaboró un informe compuesto por dos partes y un anexo.

En la primera define las nociones jurídicas y presenta las características de la organización municipal en los Estados europeos estudiados. Lo primero que hace es señalar claramente los distintos significados de la noción de competencia, distinguiendo entre potestad (power) y competencia, siendo la primera la expresión de una capacidad y un poder de actuación, que proceden de la Constitución o de la ley, y la segunda la expresión que designa el objeto al que se aplica, y/o el marco de acción en el que se ejerce esa potestad. La palabra competencia se utiliza en plural para designar los objetos o las materias sobre las que se ejerce ese poder de actuación, y en singular para designar sus límites jurídicos, es decir, para expresar la capacidad de la autoridad titular de la competencia. Este último sentido es el que usa como base de comparación en su trabajo.

Para MARCou sólo se puede definir completamente una competencia por su objeto (competencia material), las potestades destinadas a su ejercicio (una facultad o una obligación) y los medios necesarios para su aplicación, así como por el titular de esa competencia. La autonomía local es una libertad y el grado de libertad con el que esa competencia se ejerza, depende sobre todo de las potestades y los medios, sin ellos sólo es un agente de la autoridad superior en el ejercicio de la competencia material que se le atribuye.

En cambio el tema de la determinación del titular de la competencia le parece a MARCOU menos importante. En la mayoría de los países europeos se considera que es la entidad local la titular de la competencia, ejercida por sus órganos. En otros países, la autonomía local sólo la poseen los órganos que la ejercen.

En lo que respecta a las características de la organización municipal en los países estudiados, señala que a pesar de la gran variedad de instituciones municipales existen unos rasgos comunes entre todas ellas y que estos rasgos son indiferentes a la naturaleza del Estado (unitario, federal o de autonomía regional), que son: 1) El principio de autonomía local es reconocido por la Constitución o por la ley. Es este principio el que autoriza a reconocer una dimensión política, y no solamente administrativa a las instituciones municipales; 2) Se reconoce la vocación general de la competencia local. Cualquiera que sea la formulación que tome, la cláusula general de competencia es siempre indeterminada porque es una cláusula de libertad, más que un principio de atribución de competencia; 3) Las competencias siempre son definidas por la ley; 4) Las autoridades locales disponen de una potestad reglamentaria para el ejercicio de sus competencias; 5) Se les reconoce siempre la potestad de establecer ciertos tributos. Aunque la tendencia general ha sido la erosión de esta potestad en beneficio de la participación en el producto de impuestos nacionales, o más raramente regionales, y de transferencias presupuestarias; 6) Siempre existe un control de legalidad; y 7) Siempre hay procedimientos que garantizan el derecho a la libre administración de las entidades locales. Esta protección varía de unos países a otros, pudiendo distinguirse tres situaciones: en los que la protección se confía al juez constitucional, en los que corresponde al juez administrativo y aquellos en los que corresponde al juez ordinario.

Pero también desde el punto de vista de la organización municipal existen diferencias que según MARCOU son pertinentes para el análisis de las competencias. Los criterios de diferenciación son: 1) La fragmentación o la integración funcional; 2) La unidad o dualidad de la organización municipal en función de la organización urbana. Existe una tendencia a la diferenciación de los estatutos de las ciudades muy grandes, bien para tener en cuenta la función de la ciudad capital, bien para tratar de responder al desafío de la gestión de las grandes ciudades ${ }^{5}$; 3) La aplicación o ausencia de una reforma territorial; y 4) El porcentaje que representan los gastos públicos locales en el PIB. Se pueden distinguir tres grupos entre los países estudiados6: los que se sitúan alrededor del $5 \%$ (Portugal, España e Italia), los que se acercan al $10 \%$ (Alemania, Francia, Hungría, Reino Unido, Polonia y los Países Bajos) y

\footnotetext{
${ }_{5}^{5}$ En España se acaban de aprobar leyes sobre el régimen especial de Barcelona y el régimen de capitalidad especial de Madrid.

${ }^{6}$ Estos datos reflejan el peso económico de las funciones de gestión que las entidades locales garantizan, pero no reflejan exactamente la amplitud de las competencias ejercidas ni indican un grado más o menos amplio de autonomía en función del volumen de los gastos públicos locales; ya que todo depende de las condiciones en que se gestionen esos gastos.
} 


\section{RECENSIONES}

los que superan el 20\% (Suecia y otros países nórdicos).

La segunda parte la dedica a la comparación de los sistemas de competencias locales y es sobre todo de carácter metodológico. Para MARCOU la finalidad de la comparación es apreciar el grado de libertad de que disponen las entidades locales en su acción, teniendo en cuenta las relaciones que necesariamente mantienen con la administración del Estado o con las entidades regionales.

Comienza haciendo un balance de las potestades y de los medios de que disponen las entidades locales, diferenciando entre dos clases de informaciones: las que caracterizan el contexto institucional en el que actúan las entidades locales (tamaño, la existencia de servicios territoriales y el control sobre los actos) y las que reflejan los medios de acción de las entidades locales (potestad reglamentaria, libre elección de la organización y del modelo de gestión de los servicios, potestad tributaria y cooperación contractual con el Estado o la región).

Las informaciones referentes al contexto institucional permiten apreciar los factores que influyen en la libertad de acción de las entidades locales: los países con grandes entidades locales disponen de mayores capacidades administrativas; hay distintas modalidades de control sobre los actos propios de las entidades locales y; en todos los países la administración territorial comporta una red más o menos densa de servicios de las administraciones del Estado o de las regiones.

En lo que se refiere a los medios de acción de las entidades locales señala que: el titular de la potestad reglamentaria puede variar según los países: puede ser la asamblea deliberante (caso general) o el ejecutivo; la autonomía que tienen las entidades para organizarse y para elegir las formas de gestión de los servicios públicos de su competencia experimenta a veces excepciones para servicios determinados (Francia, Hungría) o esta regulada por ley (Italia, Reino Unido); las situaciones son más divergentes en lo que respecta a la potestad tributaria, pero sobre el fondo de una tendencia común: la erosión de esta potestad (solamente Italia constituye una excepción) y; existe una cierta difusión de las relaciones contractuales entre las autoridades públicas para organizar su cooperación y coordinar sus actividades, teniendo estos contratos generalmente por objeto programas o proyectos y financiaciones conjuntas ${ }^{7}$.

Después compara los ámbitos de competencia materiales de las entidades locales ${ }^{8}$, estableciendo una especie de gran clasificación que MARCOU denomina «perfiles de competencia», bastante amplios pero que permiten clarificar el panorama de la acción pública de los gobiernos locales. Son cuatro perfiles (Económico, Social, UrbanismoMedio Ambiente y Policía-Orden Público) que pueden agrupar competencias muy diversas y de muy distinta intensidad y que pueden ser más o menos marcados según los países.

Pero a pesar de la diversidad de competencias locales, el autor nos ofrece una serie de materias que podrían considerarse un mínimo común denominador competencial que estaría formado por: urbanismo, concesión de ayudas sociales y gestión de establecimientos sociales dirigidos a algunas categorías de población, infraestructuras viarias y transportes públicos, construcción y mantenimiento de centros escolares y desarrollo económico. No están en esta lista, aunque puede sorprendernos, ni el suministro de agua ni la vivienda social ${ }^{9}$.

Finalmente, pone en conexión las competencias materiales con las potestades y los medios para presentarnos las diferentes formas de relación y control que se establecen entre las entidades locales y las autoridades superiores, para poder con ello apreciar, en cada caso, en qué medida determina el grado de libertad de las entidades locales.

En lo que se refiere a los modos generales de control estudia tres cuestiones: las relaciones entre controles sectoriales y control general de legalidad ${ }^{10}$, las relaciones entre competencias propias y delegadas ${ }^{11}$ y las relaciones entre perfil de competencia y control de las finanzas locales por el poder central ${ }^{12}$.

\footnotetext{
${ }^{7}$ Donde existe la regulación más precisa es en Italia y Portugal, siendo en estos dos países junto con Francia donde están más extendidos.

${ }^{8}$ La clasificación más extendida de las competencias es la de competencias propias y delegadas. La segunda clasificación es la que distingue competencias obligatorias y facultativas (esta clasificación no se corresponde con la anterior).

${ }^{9}$ Esta competencia ha ido perdiendo progresivamente peso por reformas de cariz claramente privatizador tanto de la construcción como de la gestión e incluso de la titularidad de las viviendas.

${ }^{10}$ El modo normal de control parece ser el de legalidad de derecho común.

${ }^{11}$ Hay un mayor control de las competencias delegadas.

${ }^{12}$ Mientras que la evolución de las transferencias del Estado ha ido en la dirección de la globalización de las transferencias, a fin de dar a las entidades locales una mayor autonomía de gasto, se ve reaparecer, en numerosos países, subvenciones finalistas.
} 
Concluye señalando que el estudio de los municipios en un sector determinado debe tener en cuenta tanto la forma de financiación de sus competencias como la posición de los municipios en el contexto de las relaciones intergubernamentales que impone el marco institucional.

El informe incluye al final un anexo con los análisis de todos los países que forman la base del estudio, para facilitar al lector un mejor conocimiento de las características propias de cada país y permitirle una lectura crítica del informe.

Por último, creo que hay que agradecer al profesor Rodríguez Álvarez y al Servicio de Publicaciones del Ministerio DE ADMINISTRACIONES PúBLICAS la traducción de este informe que esta lleno de sugerencias y de análisis muy prácticos que permiten introducirse en un terreno fundamental para el futuro de la autonomía local a todas las personas interesadas en estas cuestiones. El libro les facilitará la eliminación de una serie de tópicos e informaciones incorrectas muy difundidas sobre las competencias de las entidades locales en Europa y lo hará de una forma clara, sencilla y pedagógica.

Y ello en un momento en que en nuestro país se esta terminando de elaborar el futuro marco normativo básico de autonomía local que, como el libro, tiene a las competencias como centro del análisis, pues no podemos olvidar que las competencias son, en definitiva, el elemento objetivo de la autonomía local, y los ámbitos en los que los ciudadanos identifican la acción del poder local y, por lo tanto, la manifestación más clara de su fortaleza o debilidad en el andamiaje institucional de cualquier Estado.

Carmen PINEDA NeBot

\section{Jessica MaKowiak, Esthétique et} Droit. Editorial: L.G.D.J. Librairie Générale de Droit et de Jurisprudence, E.J.A., 2006.

La presente obra trata sobre la Estética y el Derecho, aunque en realidad es un libro sobre la Estética y el Derecho administrativo, ya que este problema de la Estética tiene ante todo una importancia desde el punto de vista del Derecho Público y, en particular, desde el punto de vista del Derecho urbanístico.

La autora, no podía ser de otra forma, empieza el libro diciendo que el Derecho parece ser una realidad objetiva y, en cambio, la Estética parece ser una realidad predominantemente subjetiva.
El libro comienza haciendo un repaso histórico sobre las posturas filosóficas acerca de la Estética, en especial, en primer lugar, acerca de los autores clásicos de la Antigüedad griega. Desde estos planteamientos salta al s. XVIII, citando a Baumgarten y a Kant en el contexto de lo que la autora denomina «el apogeo de la idealización: la hipótesis de una unión entre lo justo y lo bello». Según esto una idea histórica importante sería la posibilidad de relacionar la idea de justicia con la idea de belleza.

En esta evolución histórica es interesante observar cómo tanto la Estética como el Derecho consiguen emanciparse de otras realidades superiores en las cuales se encontraban históricamente inmersos. Acontece el positivismo jurídico, la Estética deja de ligarse necesariamente a la belleza ideal. Destacable es el esfuerzo hecho en este libro por informar acerca de la consagración de la Estética como una realidad autónoma. Asimismo, en este proceso histórico habría que destacar la progresiva consagración o formación del principio de libertad de los artistas como creadores de Estética, en definitiva.

Como es obligado al tratar este tema desde un punto de vista jurídico, la presente obra busca y encuentra testimonios jurídicos, incluso jurisprudenciales y normativos, que pongan de manifiesto cómo el Derecho ha reflejado bien en la jurisprudencia bien en el articulado normativo realidades estéticas, y en este contexto se fija la autora, por ejemplo, en una Ley de 1911 que completa un Decreto de 1852 relativo a las perspectivas monumentales. Las perspectivas monumentales, según la autora, por tanto en el contexto del patrimonio histórico, serían un testimonio claro acerca de cómo el Derecho se hace eco acerca de los temas estéticos.

Otra referencia la encuentra la autora en materia de propiedad intelectual. El Derecho regula esta materia de la propiedad intelectual sobre todo a través de las ideas de originalidad y la novedad como nociones o conceptos.

Pero es en torno al Derecho regulador de la cultura y del arte donde esta obra aporta mayores ejemplos de la interconexión entre la Estética y el Derecho. Hay ocasión en el presente libro incluso para tratar dimensiones estrictamente prácticas, como las económicas y las sociales, ilustrando la autora acerca de los contenidos de los contratos de servicios estéticos o los contratos de venta relativos a los productos de belleza y de otros contratos firmados por profesionales cuya actividad se relaciona con la Estética.

Según la autora, el Derecho ha procurado una protección preventiva y defensiva de la Estética 
evitando que este valor se desvirtúe ante las amenazas que padece. Desde el punto de vista del Derecho es obligado tratar la libertades «de espíritu» (dice la autora), es decir, libertades cinematográficas o artísticas, incidiendo en el derecho incluso constitucional de libertad del artista.

En el Derecho francés, la Ley de Arquitectura de 3 de enero de 1977 y su afirmación en el artículo 1 -según el cual «la arquitectura es una expresión de la cultura, proclamando al mismo tiempo la creación arquitectónica como valor fundamental de interés público»- consigue erigirse en un punto de referencia fundamental para la materia que nos ocupa.

De forma interesante, la autora trata de conciliar la belleza natural y la belleza artificial desde el punto de vista de la Estética. Este debate es una discusión clásica que nos recuerda aquel debate existente entre naturalistas y simbolistas, típico a finales del siglo XIX, por ejemplo, entre Balzac por un lado y Villiers por otro lado.

El libro se ocupa también del paisaje como concepto que da juego para avanzar en la investigación en materia estética desde el punto de vista jurídico, con numerosos testimonios normativos y jurisprudenciales.

Seguidamente se analizan en la obra los mecanismos que aporta el Derecho para proteger la Estética frente a las inmisiones visuales contrarias a este tipo de conceptos culturales estéticos, tales como las privaciones de vistas o de contemplaciones.

Otras reflexiones las dedica la autora a la democratización de la Estética, es decir, un ensayo que pone en relación la democracia como valor y la Estética.

En el contexto del concepto fundamental de orden público la autora trata también algunas referencias menores a la Estética en el ámbito del Derecho urbanístico. El libro recoge aquellas concepciones de «la Estética decretada», es decir, aquellos que apasionados posiblemente por la Estética llegan a un planteamiento jurídico no del todo aceptable (y este es el problema de fondo de la Estética), ya que este valor no puede conseguirse a costa de sacrificar los derechos de los individuos. Otro concepto relacionado con la Estética sería el de la Estética controlada, ya no decretada.

En todo caso, parece conveniente que se publiquen estudios como el presente, ya que tradicionalmente este concepto de la Estética ha causado cierta incomodidad para los juristas en general.
Habrá que seguir el desarrollo de esta interrelación entre Derecho y Estética que tanto interesa para el Derecho urbanístico, en especial, así como para el Derecho en general, desde el punto de vista de la Filosofía del Derecho u otras disciplinas posibles.

\author{
SANTIAGo GoNZÁlEZ-VARAS IBÁÑEZ \\ Catedrático de Derecho Administrativo \\ Universidad de Alicante
}

\section{Manuel José Domingo Zaballos (di- rector), Práctica de Administración local: formularios y documentos. Editorial Tirant lo Blanch. Valencia, 2006.}

Damos noticia, y contribuimos así a su conocimiento y difusión, de una reciente obra que lleva por título «Práctica de Administración local: formularios y documentos». Su vocación es, en efecto, eminentemente práctica. Consta aquella de dos amplios volúmenes o tomos, con magnífica edición y presentación, donde se abordan las distintas esferas de actuación de la Administración local, así como de un CD-Rom auxiliar.

Tal como se indica en la presentación «esta obra colectiva trata de facilitar la práctica administrativa en nuestros entes locales. Nada más pero nada menos. De ahí que, a salvo de alguna nota aclaratoria, no incorpore «introducciones»o «comentarios», que dejamos para otro tipo de obras existentes o por publicar». El trabajo se ha hecho pensando en quienes desarrollan su labor como profesionales o cargos electivos locales, pero sin olvidar que, comúnmente, la maquinaria administrativa se activa porque lo interesan los ciudadanos - por escrito, claro- directamente o a través de abogados, gestores... de manera que también incorpora el libro escritos de particulares, sigue diciendo la presentación.

Siendo esto así, esta reseña quiere, no obstante, apuntar algunos puntos meritorios de esta obra que acaso no correspondían, por pura modestia, ser destacados por el coordinador del libro, pero que no pueden tampoco del todo obviarse. Primeramente, en varios formularios que recoge esta publicación se exponen, inevitablemente, las regulaciones que sirven de fundamento jurídico a la toma de decisiones y, por tanto, a los distintos escritos, resoluciones o sobre todo informes que nos aportan los autores. Es decir, incluso desde un punto de vista metodológico, cabría decir que esta obra también aporta conocimiento del Dere- 
cho, aunque sea con un método distinto del método científico, que no se pretende. Más bien, es interesante observar la «aplicación» concreta y práctica de las doctrinas legales a los informes municipales para resolver problemas jurídicos. Así pues, la obra aporta conocimiento jurídico no sólo formal sino también sustantivo.

Por otro lado, es destacable la paciente labor de los autores y meritorio el número de horas de trabajo que conlleva realizar una obra de estas características, máxime cuando es inherente a toda labor de documentación el esfuerzo adicional de tener que completar originalmente la presentación de algunos de los informes necesarios durante la tramitación de un procedimiento. Asimismo, la selección del ejemplo práctico completo (por ejemplo en materia de permutas de bienes), para que el lector tenga a disposición un ejemplo ordinario y pacífico, lejos de la complicación que planteará la casuística, requiere sensibilidad jurídica.

En este sentido, también ha de destacarse el logro de uniformidad, no reñido con la diversidad temática, pues, desde este último punto de vista, no queda tema jurídico local sin abordar en esta obra. Más bien, se va haciendo un repaso sobre las distintas esferas de actuación de los Ayuntamientos para aportar documentos en cada una de ellas: bienes, contratación, personal, urbanismo, etc. Se guarda la proporción, ya que no domina una materia sobre otra. Asimismo, se consiguen tratamientos diferenciados cuando la materia puede aconsejarlo, como por ejemplo incorporando distinta reglamentación sobre los Consejos Escolares Municipales según se trate de municipios de un solo centro escolar o con varios.

Otro mérito, o al menos reto conseguido, sería la utilidad de la obra en toda España, lo que no es fácil ante la complicación y traba que para ello representa actualmente el sistema de las Comunidades Autónomas siempre preocupado de establecer matices o distinciones frente a un sano y uniforme régimen jurídico general nacional. Se evitan de esta forma alusiones a regiones o lugares concretos y alcanza el libro validez general.

Un factor característico de nuestro tiempo es el de las reformas incesantes legislativas. En este sentido, «Práctica de Administración local: formularios y documentos» presenta una versión actualizada de los distintos informes y documentos.

Se están publicando recientemente distintas obras generales de distinta procedencia y cabe esperar que la labor de asesoramiento mejore dentro de unas Corporaciones locales, abrumadas de trabajo por un lado y desasistidas por otro. Vieja reclamación ésta en la que no vamos a profundizar ahora, a salvo de relacionarla con la bondad de publicaciones o acciones que contribuyan a poner remedio a este problema. La Editorial Tirant lo Blanch Valencia 2006, siempre atenta a estas necesidades, cubre de manera ejemplar esta necesidad a través de obras científicas y otras de otro tipo, como la presente.

Los autores del libro ahora reseñado son todos ellos consumados especialistas del mundo local. El coordinador del libro, en particular, actualmente magistrado de lo contencioso-administrativo en el Tribunal Superior de Justicia de la Comunidad Valenciana, D. Manuel José Domingo Zaballos es consumado especialista en este área, como lo corrobora su previa dedicación al Derecho local en muy diversos campos.

Evidentemente es la virtualidad práctica de la obra la vertiente dominante y lo que le convierte en un punto de referencia actual para los prácticos del Derecho pero también, como decía, para los propios estudiosos del mundo jurídico o administrativo local. La labor práctica del Derecho cumple su genuina función en el mundo local, tan apegado a las necesidades concretas de los individuos y es una labor, cuando se hace bien, que representa una especial complejidad al suponer la aplicación del conocimiento teórico de las leyes a casos concretos.

¡La práctica jurídica, algo tan fácil para quien la conoce, y tan difícil para quien se encuentra lejano a ella. O, dicho de otra forma, algo cuya importancia relegamos cuando la dominamos, pero que mitificamos cuando no la conocemos!

Santiago GonZÁLEZ-VARAS IBÁÑEZ Catedrático de Derecho Administrativo Universidad de Alicante

\section{Santiago GonZález-VARAS IbáÑEZ (coordinador), Nuevo Derecho de Aguas. Editorial Civitas. 2007.}

El libro «nuevo Derecho de aguas» representa una de las aportaciones más recientes y sobresalientes del Derecho de aguas en España.

La publicación citada se patrocina por el Ente Público del Agua, una nueva entidad empresarial de la Región de Murcia (regulada por la Ley 4/ 2005 , de 14 de junio de la citada Región). Precisamente en parte esta obra consigue explicar los nuevos desarrollos del Derecho de aguas y uno 


\section{RECENSIONES}

de estos desarrollos o novedades es la creación de esta entidad para la mejor solución de los problemas del agua, en especial en el contexto de la desalación.

El libro realiza una presentación o estudio general y particular de este Ente (conocido abreviadamente como EPA), cuyo conocimiento es imprescindible hoy día por todos aquellos estudiosos del Derecho de aguas en nuestro país. El libro contribuye a su difusión. De hecho, dicho libro ha conseguido hasta el momento que dicho EPA sea ya bien conocido allende nuestras fronteras, por ejemplo en Chile, país donde también se desarrollan interesantes estudios sobre esta materia.

Sobre el EPA la obra lleva a cabo un exhaustivo estudio sobre su régimen jurídico-administrativo, superando la especial complejidad que presentan estas entidades que se mueven a caballo entre el Derecho público y el Derecho privado. También se estudian las distintas fuentes normativas de esta entidad. Este aspecto de la difusión del EPA, en el contexto general de la doctrina de aguas de España y otros países, ha sido apreciado por esta institución, el EPA pues no en vano ha promocionado la obra y su logotipo aparece en la portada del libro.

El autor que se dedica a desarrollar esta materia, Santiago González-Varas estudia este Ente no escatimando subrayar las novedades y aportaciones de este Ente, a pesar de que es inherente la polémica a este tipo de instituciones relacionadas con el agua, tema siempre complejo, y relacionadas con entidades de difícil encaje entre el Derecho público y privado.

El citado profesor González-Varas, catedrático de Derecho administrativo de Alicante activo en las zonas de Valencia y Murcia, ha dirigido además la obra que reseñamos. Esta labor de coordinación representa un notable esfuerzo, como es sabido. Es preciso perfilar los temas del libro, es preciso buscar a los mejores especialistas en la materia, es necesario coordinar con ellos y dialogar acerca de las líneas de trabajo y temáticas, etc, etc.

La concreta problemática del agua en la región de Murcia deja su huella en la obra, como no podía ser de otra forma. El libro consigue finalmente trascender del típico localismo de nuestros días, alcanzando incluso una dimensión internacional, pero qué duda cabe que la problemática de la Región de Murcia, marcada por la escasez de recursos hídricos, define la primera contribución del libro. Pensamos que, en este sentido, la notable la- bor de las instituciones de Murcia, en aras de procurar dar soluciones al problema de la escasez de agua, queda bien destacada por González-Varas. La desalación, en particular, se estudia con detalle por C. Jiménez Shaw, Díaz-Romeral Gómez en uno de los capítulos del libro.

La parte, en concreto, del citado Profesor ocupa ochenta hojas a un espacio, la más extensa del libro, pero este libro recoge además las aportaciones de numerosos autores de España y de otros países y pensamos que la crítica ha de ser favorable a esta publicación. Por otra parte, no puede pasar desapercibido este libro en el contexto de los derechos del agua.

Consigue así erigirse esta publicación en un Tratado de Derecho de aguas. Siguen al estudio referido contribuciones generales («el agua como derecho humano» del catedrático Demetrio Loperena), pero también otras más particulares, por ejemplo las concernientes al Derecho europeo y el agua (así, el trabajo de D. Ordoñez Solís o el de J. Agudo González o L. Casado Casado). El aspecto organizativo y competencial también ocupa un espacio relevante del libro (mancomunidades, consorcios...), así como los temas más clásicos de las concesiones de aguas o el régimen sancionador.

Asimismo, no podemos dejar de citar los capítulos sobre vertidos y reutilización del agua que brillantemente analizan Joaquín Melgarejo, Ruiz de Apodaca, todos ellos autoridades y especialistas de gran prestigio en la materia de aguas, al igual que los demás autores no citados en esta reseña pero que participaron en esta obra y que lo hicieron además en alguna conferencia sobre aguas por invitación del coordinador del libro, durante 2006, lo que supone un esfuerzo añadido en el presente contexto.

Son destacables además las contribuciones de autores extranjeros, argentinos, costarricenses, chilenos, norteamericanos, que consiguen otorgar una dimensión y difusión comparada al presente libro.

Es decir, dicho libro consigue ser en estos momentos la obra más completa y más autorizada sobre el Derecho de aguas, publicada de forma lujosa en una editorial de especial prestigio y por autores especialistas en la materia del agua. De ahí que sea recomendable este libro, y en general la materia del agua ha de merecer y está mereciendo ya a mi juicio una creciente atención.

Inmaculada DE LA FUENTE CABERO

Dra. Europea, abogada y LLMM en Dresden 